OPEN ACCESS

Edited by:

Kerrie Leanne McDonald,

University of New South Wales,

Australia

Reviewed by:

Kamalakannan Palanichamy,

The Ohio State University Columbus,

United States

Maria Caffo,

University of Messina, Italy

*Correspondence:

Udo S. Gaipl

udo.gaip/@uk-erlangen.de

tThese authors have contributed equally to this work and as senior authors to the study.

Specialty section: This article was submitted to Neuro-Oncology and Neurosurgical Oncology, a section of the journal

Frontiers in Neurology

Received: 10 April 2017 Accepted: 09 June 2017

Published: 23 June 2017

Citation:

Rühle PF, Goerig N, Wunderlich R, Fietkau R, Gaipl US, Strnad A and

Frey $B$ (2017) Modulations in the

Peripheral Immune System of Glioblastoma Patient Is Connected to Therapy and Tumor Progression-A

Case Report from the IMMOGLIO-01 Trial.

Front. Neurol. 8:296

doi: 10.3389/fneur.2017.00296

\section{Modulations in the Peripheral Immune System of Glioblastoma Patient Is Connected to Therapy and Tumor Progression-A Case Report from the IMMO-GLIO-01 Trial}

\author{
Paul F. Rühle', Nicole Goerig', Roland Wunderlich ${ }^{1,2}$, Rainer Fietkau', Udo S. Gaip/1*t, \\ Annedore Strnad ${ }^{1 t}$ and Benjamin Frey ${ }^{1 \dagger}$ \\ 'Department of Radiation Oncology, Universitätsklinikum Erlangen, Friedrich-Alexander-Universität Erlangen-Nürnberg, \\ Erlangen, Germany, ${ }^{2}$ Research Unit of Radiation Cytogenetics, Helmholtz Center Munich, Neuherberg, Germany
}

Immune responses are important for efficient tumor elimination, also in immune privileged organs such as the brain. Fostering antitumor immunity has therefore become an important challenge in cancer therapy. This cannot only be achieved by immunotherapies as already standard treatments such as radiotherapy and chemotherapy modify the immune system. Consequently, the understanding of how the tumor, the tumor microenvironment, and immune system are modulated by cancer therapy is required for prognosis, prediction, and therapy adaption. The prospective, explorative, and observational IMMO-GLIO-01 trial was initiated to examine the detailed immune status and its modulation of about 50 patients suffering from primary glioblastoma multiforme (GBM) or anaplastic astrocytoma during standard therapy. Prior to the study, a flow cytometry-based assay was established allowing the analysis of 34 immune cell subsets and their activation state. Here, we present the case of the first and longest accompanied patient, a 53-year-old woman suffering from GBM in the front left lobe. In context of tumor progression and therapy, we describe the modulation of the peripheral immune status over 17 months. Distinct immune modulations that were connected to therapy response or tumor progression were identified. Inter alia, a shift of CD4:CD8 ratio was observed that correlated with tumor progression. Twice we observed a unique composition of peripheral immune cells that correlated with tumor progression. Thus, following up these immune modulations in a closely-meshed manner is of high prognostic and predictive relevance for supporting personalized therapy and increasing therapy success.

Clinical Trial registration: ClinicalTrials.gov, identifier NCT02022384 (registered retrospectively on 13th of December, 2013).

Keywords: glioblastoma multiforme, radiochemotherapy, immunophenotyping, immune status, liquid biopsy, personalized medicine

Abbreviations: GBM, glioblastoma multiforme; CT, chemotherapy; RT, radiotherapy; RCT, radiochemotherapy; TMZ, temozolomide (brand name: Temodal); MRI, magnetic resonance imaging; VEGF, vascular endothelial growth factor; DC, dendritic cells; pDC, plasmacytoid DC; mDC, myeloid DC. 


\section{INTRODUCTION}

Glioblastoma multiforme (GBM) is the most common primary malignant brain tumor which is very aggressive and almost always recurrent $(1,2)$. Despite the improvements in the multimodal therapy including surgery, radiotherapy (RT), and chemotherapy (CT), GBM is still associated with a poor median survival of only 13-16 months $(1,3)$ and a 5-year survival rate below $5 \%(1,4,5)$. Therefore, further therapy options, such as immunotherapy (6-8), are desperately needed. Even though access to the brain is highly regulated by the blood-brain barrier, immunotherapies might be very beneficial in GBM treatment as today it is commonly accepted that leukocytes migrate into the brain (9). Furthermore, it has been demonstrated that microenvironment of GBM drives chemo- and immunotherapy resistance (10) and that the dynamics of interactions of immune cells that pass the blood-brain barrier with glioblastoma cells contribute to glioblastoma pathogenesis and might serve as basis for therapy improvements (11).

Highly important for the successful application of immunotherapy together with radiochemotherapy (RCT) is the right time point for its inclusion and a previous estimation of individual therapy responses. Therefore, we initiated the IMMO-GLIO-01 study. The goal of this prospective, explorative, and observational study has been a detailed longitudinal evaluation of the immune status from patients suffering from GBM grade IV or anaplastic astrocytoma grade III in course of a standard RCT. Eventually, immune biomarkers should be defined that are connected to individual therapy response for translation into clinic. For this, liquid biopsies are mandatory since solid biopsies can only be taken at limited time points and, therefore, just reflect a snapshot of the disease (12). We, therefore, previously established a flow cytometry-based assay for detailed immunophenotyping of blood allowing the identification of 37 different cell subsets circulating the periphery and the characterization of their activation status without the need of preceding cell isolation (13).

Here, we present the immunological data of the first and longest accompanied patient in the IMMO-GLIO-01 study. We show that intensive RCT does not lead to general immune suppression, but rather to distinct immune modulations and that the latter might be linked to therapy and clinical response of the tumor.

\section{METHODS}

The IMMO-GLIO-01 study (clinical trials ID: NCT02022384) is a prospective, explorative, and observational trial at the Department of Radiation Oncology at the Universitätsklinikum Erlangen and has been performed in accordance with the Declaration of Helsinki. Ethical approval was granted from the ethics committee of the University Erlangen-Nürnberg Medical School. The patient signed a declaration of consent to participate in the study and provided a written consent that the obtained data can be published as case report or as any other publication in a pseudonymised manner. Blood was drawn in a closely-meshed manner during course of tumor therapy as indicated in Figure 1

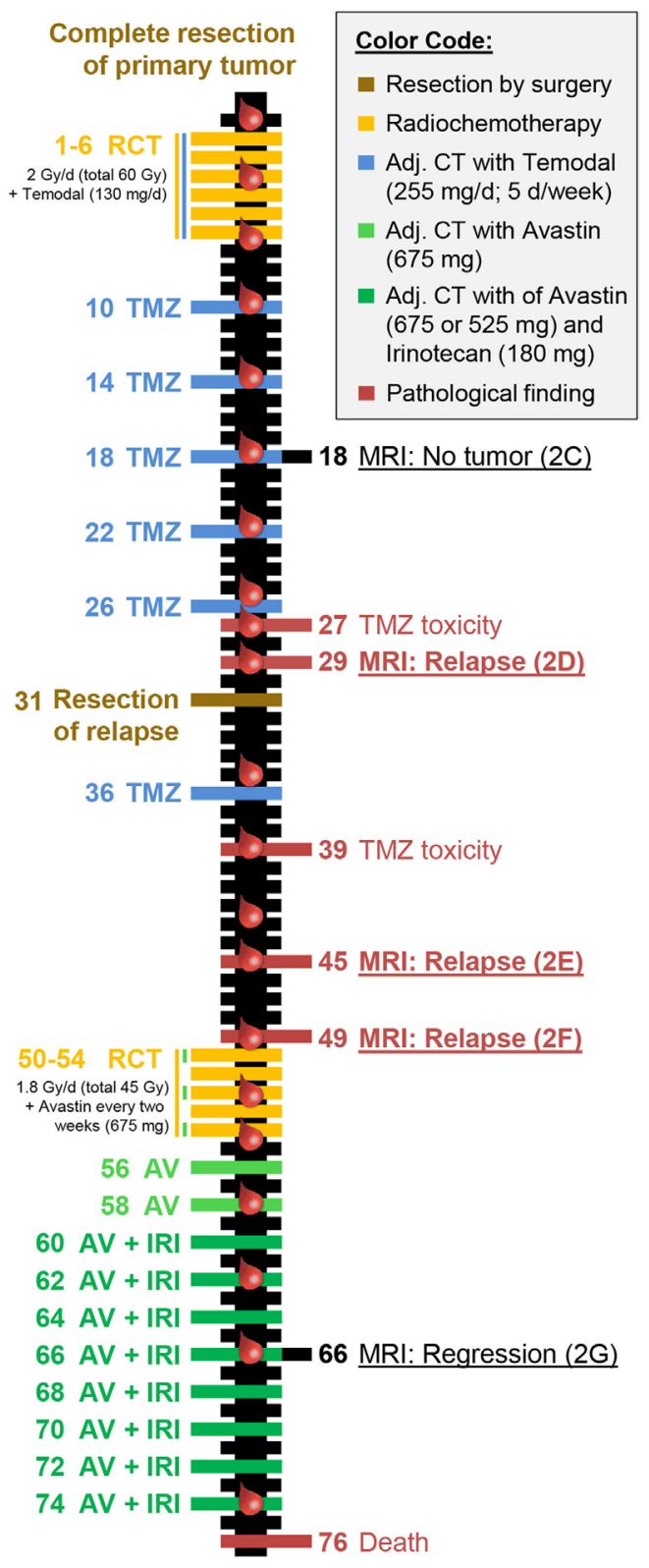

FIGURE 1 | Individual therapy schedule and clinical response of the reported patient with glioblastoma. The time line depicts treatment (left side) and clinical response (right side) including pathological abnormalities and tumor progression. All numbers stated are in weeks with zero simultaneously being the starting point of radiochemotherapy and the evaluation of the immune status (indicated by red blood drops). The colors represent treatments or therapy outcomes according to the color code presented in the top right corner. This color code was also used for data presentation in Figure $\mathbf{3}$. The magnetic resonance imaging scans directly refer to the according image in Figures 2C-G.

(red blood droplets). The blood was always taken before administration of therapeutics on that day avoiding misinterpretation by immediate short-time reactions. For all 21 blood samples, we performed detailed immunophenotyping (13). The staining and analysis of all samples were performed blinded. 


\section{PRESENTATION OF THE CASE: CLINICAL PART}

The first patient included in the IMMO-GLIO-01 study was a 53-year-old woman suffering from right temporal GBM grade IV (Figure 2A). She was recruited into the study directly after complete resection of the primary tumor (Figure 2B) and then received standard RCT according to Stupp et al. (3) (6 weeks, 60 Gy in 2 Gy fractions, daily $130 \mathrm{mg}$ Temodal (TMZ); therapy scheme presented in Figure 1). Then, therapy was continued as adjuvant CT with TMZ (255 mg/day) until the fifth course (week 26) without any neurological or pathological complications [magnetic resonance imaging (MRI) presented in Figure 2C]. But in week 27 the patient complained of loss of appetite and nausea. Further examinations revealed an increase of transaminases to grade III-IV and a hepatic steatosis. Consequently, MRI was performed (week 29) depicting signs of a relapse (Figure 2D), which was later confirmed by histological examination. The interdisciplinary tumor board decided to conduct a second surgery (in week 31) and the relapsed tumor was successfully removed. As afterward the patient did not show any further clinical complications, the TMZ therapy was pursued with reduced dose $(80 \%$, week 36). Despite dose reduction of TMZ, the transaminase levels again increased to grade III (week 39). Consequently, TMZ therapy was stopped.

The next routine MRI was conducted in week 45 ( 3 months after surgery) and week 49 and revealed a new relapse (Figures 2E,F), but again this was paired without any neurological findings. This time, the interdisciplinary tumor board decided for a conversion of therapy to Avastin (bevacizumab) targeting vascular endothelial growth factor (VEGF), which had been approved for the treatment of recurrent gliomas. The RCT in combination with Avastin (5 weeks: 45 Gy in 1.8 Gy fractions, biweekly $675 \mathrm{mg}$ Avastin) was followed by an adjuvant CT with biweekly applied Avastin (week 56 and week 58) or Avastin in combination with Irinotecan (from week 60 on). The success of this therapy conversion became visible in the next routine MRI (week 66) that showed a regression of the tumor relapse (Figure 2G). During Avastin therapy, the patient never showed any neurological or pathological complications, however, the patient died in week 76 in succession of an epileptic seizure as complication of the disease. Another fact worthy of note is that the patient did not have any infections during complete therapy and did not take any steroids, except directly after surgery.

\section{PRESENTATION OF THE CASE: IMMUNE STATUS}

The leukocyte count varied during the whole therapy, but never strikingly dropped below the normal range (Figure 3A). This emphasizes that the immune system is not destroyed during intensive anticancer RCT of glioblastoma. Yet, the leukocyte count decreased following RT in both applications (yellow bars), but recovered afterward. Besides, we observed a strong increase in the leukocyte count after discovery of the second relapse (red bars, week 45 and week 50), which especially was caused by neutrophils (Figure S1A in Supplementary Material).

The investigation of $\mathrm{T}$ cells revealed a strong temporary decrease following RT from 1,664 to 753 cells/ $\mu$ l (second RT: 1,488 to 737 cells/ $\mu \mathrm{l}$ ), but they recovered fast and then varied without distinct patterns during the remaining therapy (Figure S1B in Supplementary Material). In contrast, we found a connection between therapy and $\mathrm{T}$ cell subsets, which is presented as ratio of $\mathrm{CD} 4^{+} \mathrm{T}$ helper versus $\mathrm{CD} 8^{+}$cytotoxic $\mathrm{T}$ cells (Figure $3 \mathrm{~B}$ ). During administration of TMZ (blue dots), a strong shift toward cytotoxic T cells was observed. This was reversed in both relapse situations (red dots) and not observed following Avastin therapy (green dots). In this context, RT (yellow dots) might be inhibitory on or delaying the TMZ effect as during initial RCT (combined with TMZ) a small decrease in the CD4:CD8 ratio was identified. During the second RCT (combined with Avastin) no influence of RT on the CD4:CD8 ratio was seen.
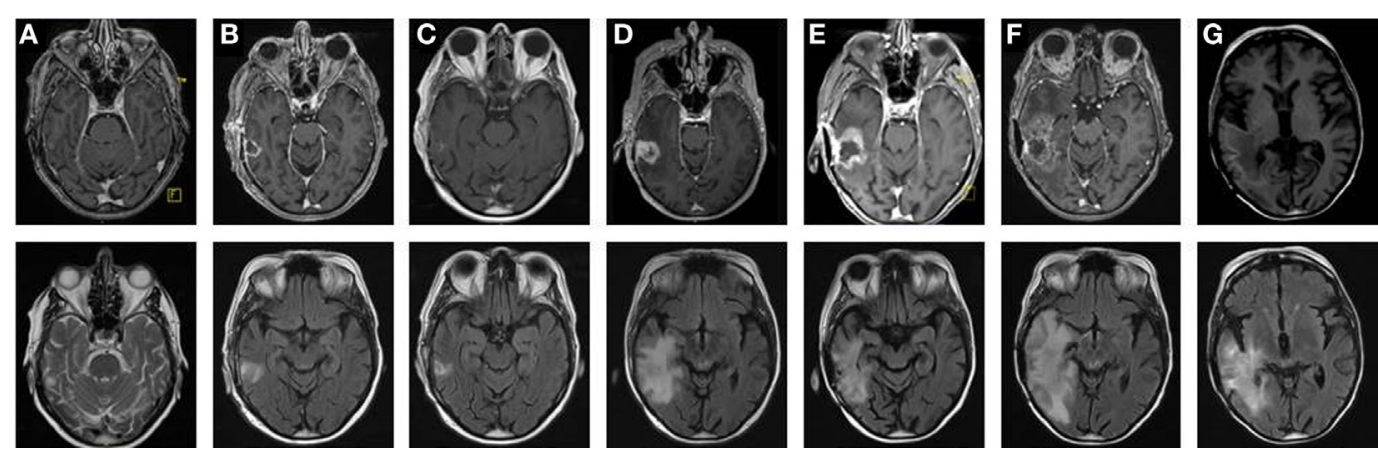

FIGURE 2 | Tumor progression identified by consecutive magnetic resonance imaging (MRI) scans reveals transient therapy success and two relapses. MRI scans showing T1- (upper panel) and T2-weighted images (lower panel) of a glioblastoma multiforme in the right temporal lobe, from primary diagnosis through the different stages of tumor progression. After primary diagnosis (A) and subsequent complete resection (B), standard concomitant radiochemotherapy (RCT) followed by adjuvant chemotherapy with Temodal (TMZ) according to Stupp et al. was performed. The first follow-up MRI ( 3 months after RCT) showed no signs of tumor recurrence (C). Two months later, an MRI was performed due to TMZ cytotoxicity. There were hints of tumor relapse (D), which was histologically confirmed after the lesion had been completely removed by surgery. Yet only 2 (E) and 3 months (F) after this second resection, tumor progression was first suspected and then confirmed. Thus, a conversion of therapy was decided, the therapy of choice being secondary radiotherapy combined with Avastin. This led to tumor regression as observed during the 3-month follow-up during aftercare with Avastin and irinotecan (G). 

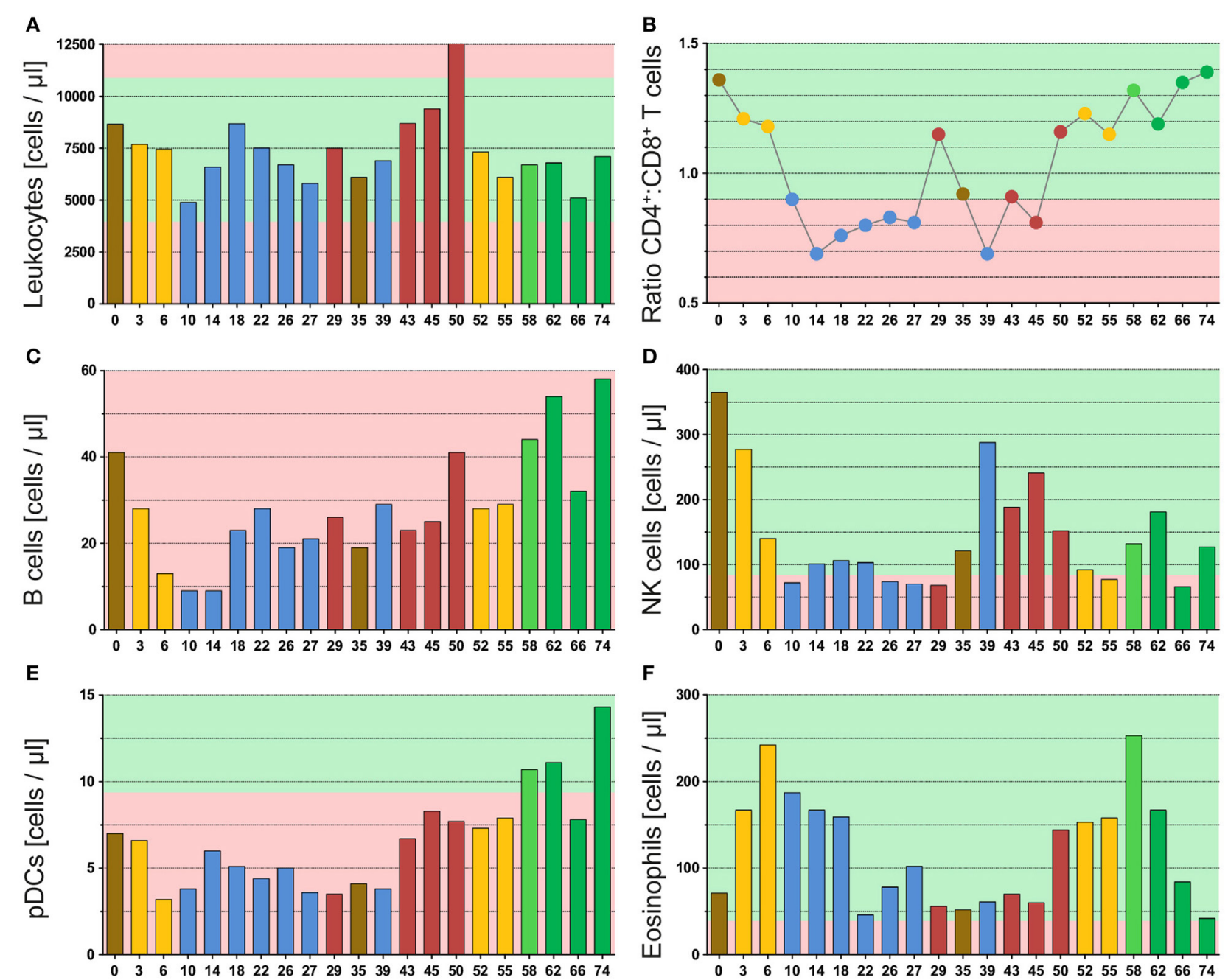

FIGURE 3 | Multimodal therapy of glioblastoma has high impact on immune cells of the peripheral blood. The graphs of (A) and (C-F) display the absolute cell counts of immune cells or immune cell subsets in the peripheral blood, and in (B), the ratio from CD4+ helper T cells to CD8 ${ }^{+}$cytotoxic T cells is displayed. All immune cells were determined by a multicolor flow cytometry-based assay and directly detected in whole blood samples. The blood was always drawn prior to administering the drug or radiotherapy on the respective day. The color code represents the last applied therapy or pathological finding (red) and was applied according to Figure 1. The green background marks normal values and the red one deviation from it.

The Avastin therapy improved the B cell count (Figure 3C: green bars), which was very low during the complete observation period $(<40$ cells $/ \mu \mathrm{l})$ and did not rise before Avastin administration. As observed for T cells, the B cell count dropped following initial RCT (from 41 to 13 cells/ $\mu \mathrm{l}$ ), but recovered later on, even this required more time. In contrast to T cells, the second RCT in combination with Avastin had only marginal impact on the $\mathrm{B}$ cell count suggesting a protective or beneficial role for Avastin on B cells.

The initial RT, likewise, had a very strong effect on NK cells which dropped from 365 to 140 cells/ $\mu$ l and remained fairly low during the complete period of adjuvant TMZ administration (Figure 3D: week 0-29). Following TMZ discontinuation and surgery, the NK cells increased again. Yet, the second RT induced a strong decline from 152 to 77 cells/ $\mu$ l. But in contrast to adjuvant TMZ administration, NK cells could recover during Avastin therapy (green bars) even thus they did not reach the initial level.
Also striking was the pattern of plasmacytoid dendritic cells (pDC). These immune cells also decreased during initial RCT (Figure 3E: from 7 to 3 cells/ $\mu \mathrm{l}$ ) and remained low during adjuvant TMZ administration, but increased heavily during Avastin intake (11-14 cells/ $\mu \mathrm{l})$. The pattern was very similar to that of B cells.

Furthermore, we found a surprising enhancement of eosinophils by RT, which was observed following both applications (Figure 3F: yellow bars). However, the second increase was already detected before start of RT, namely after detection of the second relapse in week 50. During that time point likewise neutrophils (Figure S1A in Supplementary Material) and B cells (Figure 3C) were conspicuously elevated.

Besides, the 29 week-time point (detection of the first relapse) was noticeable as an elevation of monocytes (Figure S1C in Supplementary Material) and neutrophils (Figure S1A in Supplementary Material) was observed. Moreover, the CD4:CD8 
ratio was extremely reversed in direction of $\mathrm{CD}^{+} \mathrm{T}$ helper cells in both relapse situations (Figure 3B). This suggests a connection of these immune parameters to clinical response as they correlate with tumor progression monitored by MRI.

\section{DISCUSSION}

The IMMO-GLIO-01 study was initiated to estimate whether and in particular how multimodal therapy of brain tumors (Figure 1) including RCT impacts on the systemic immune status of the patients. Immunological patterns might be discovered that do relate to therapy application, response, and/or disease status. These patterns should be useful for estimation of individual responses (Figure 2), to stratify patients, and to estimate suited time points for inclusion of further immunotherapy (14). An intensive cytotoxic therapy as applied in the treatment of high-grade gliomas should certainly cause leukopenia (15). This was also observed in the presented case, especially following RT (Figure 3A). But, all immune subsets recovered in course of therapy and some even increased. This suggests the immune system to remain functionally active, as previously described for other tumor entities (16). Moreover, the immune system seemed to contribute to tumor elimination as we found certain immune modulations to be connected to tumor progression or therapy, even though, none of the administered therapeutics was particularly targeted against any immune cell.

The most striking pattern observed was the $\mathrm{CD} 4^{+}: \mathrm{CD} 8^{+}$ratio that strongly shifted into direction of $\mathrm{CD}^{+}$cytotoxic $\mathrm{T}$ cells following TMZ administration. In the treatment of gliomas, TMZ is prescribed as it easily crosses the blood-brain barrier (17) and induces a G2-M arrest, especially in p53-mutated cells, which is true for most glioma cells. This leads to synchronization of the cell cycle in a radiosensitive phase (18). Our data suggest that TMZ additionally has an immunogenic potential supporting the stimulation of tumor killing $\mathrm{CD}^{+} \mathrm{T}$ cells in the periphery. However, this has to be proven by analyses of more patients within the IMMO-GLIO-01 trial. In fact, we measured a reversed setting during both relapse situations after the TMZ intake was stopped (Figure 3B). Yet, it remains unclear whether the decline of cytotoxic $\mathrm{T}$ cells led to a recurrence of the tumor or the progressing tumor induced a suppression of cytotoxic T cells. However, this ratio does not implicitly reflect the absolute count as the $\mathrm{T}$ cell count varied and in general was lower than before therapy. Anyhow, this distinct pattern might be beneficial for estimation of individual therapy responses and for identification of tumor progression. Besides, in vitro studies have shown that TMZ (as well as Avastin) has no negative effects on the functionality of $\mathrm{CD}^{+}$cytotoxic T cells (19) indicating not only a TMZ-related change in T cell numbers but also an enhanced antitumor immunity. In contrast, Grossman et al. (20) demonstrated that patients with a very low $\mathrm{CD} 4^{+}$ $\mathrm{T}$ cell count induced by RT/TMZ have a lowered overall survival. These early deaths were not related to infections, but to an earlier tumor progression. Therefore, a drop of CD4:CD8 ratio might be adverse when it is due to very low $\mathrm{CD} 4^{+} \mathrm{T}$ cell count $(<200$ cells $/ \mu \mathrm{l})$ and not due to an increasing number of $\mathrm{CD}^{+}$cytotoxic $\mathrm{T}$ cells. Interestingly, during the period of dropping CD4:CD8 ratio, the innate NK cells were repressed
(Figure 3D). These innate immune cells also play a major role in killing of tumor cells, but are less specific. We conclude that therapy-modified adaptive and innate immune responses are interconnected.

Besides leukopenia, RT caused an enhancement of eosinophils in both applications (Figure 3F). Eosinophilia in cancer treatment remains elusive and there are controversial reports associating it with good or bad prognosis (21-23). But these reports mainly investigated the level of eosinophils without a simultaneous evaluation of other infiltrative immune cells. In contrast, Carretero et al. (24) showed that eosinophils might indeed support tumor rejection, but only in the presence of tumor-specific $\mathrm{CD}^{+}$ $\mathrm{T}$ cells. Moreover, activated eosinophils were not destructive cells releasing their cytotoxic granules, but modulated the tumor microenvironment by chemokine production and thus improved $\mathrm{T}$ cell infiltration. In addition, it was demonstrated that activated eosinophils preferentially migrated into tumor tissues. This attraction was probably achieved by release of damage-associated molecular patterns from the necrotic tumor cells (21). We now also detected an increase in eosinophils in the periphery, which only can be detected when whole blood samples are analyzed and, therefore, might be overseen in several routinely performed assays using isolated peripheral blood mononuclear cells as biomaterial. As RT is capable of enhancing tumor necrosis (25), this could explain the peripheral rise of eosinophils observed in this case report. Taken together, an elevated number of eosinophils followed by an increase in $\mathrm{CD}^{+} \mathrm{T}$ cells might be an immune marker for a beneficial therapy response.

The role of B cells in antitumor immunity remains debatable and, like eosinophils, so far was correlated with both improved $(26,27)$ and poor prognosis $(28)$, which depends on balance of the various $B$ cell subsets within the tumor microenvironment (29). On the one hand, these may enhance antitumor responses supporting $\mathrm{CD}^{+} \mathrm{T}$ cells by cytokine secretion or antigen presentation (30). But on the other hand, infiltrative B cells might even inhibit an effective induction of cytotoxic $\mathrm{T}$ cell response $(31,32)$. Thus, in the final analysis covering all patients, a careful comparison of the $\mathrm{B}$ cell subsets and individual therapy response will have to be performed. In the presented case, we found the $B$ cells in a very low frequency, as described for many malignancies, and they did not recover until Avastin administration (Figures 1 and 3C). Avastin is an antibody designed to bind and neutralize VEGF activity. VEGF is overexpressed by most gliomas and plays an important role in angiogenesis, and initially its neutralization was believed to inhibit tumor vascularization and consequently interrupt its blood supply $(33,34)$. But more important, Avastin affects the malformed tumor vessels resulting in a normalization of them (33-35). This leads to not only reduced leakage and, therefore, lowered edema and intracranial pressure but also enhanced immune infiltration (36) as well as an influx of cytotoxic agents (35), such as irinotecan. Besides, the blocking of VEGF might also have a positive effect on dendritic cell (DC) number and maturation (37).

Irinotecan inhibits topoisomerase I and, therefore, prevents DNA from unwinding and consequently inhibits transcription and replication (38). Among others, myelosuppression and neutropenia are characterized side effects of irinotecan (39), but 
were not observed in the peripheral blood of the presented case (Figure 3). Although, here it should be stressed that direct shortterm effects were not investigated as blood withdrawals were always conducted before drug administration pursuing the detection of general immune modulations. Anyhow, irinotecan might be involved in the conspicuous decline of many subsets in week 66 (Figure 3). Besides, Avastin administration might support the elevation of B cells and, therefore, probably pushes the humoral immunity. Concurrently, the pDCs increased (Figure 3E), which has often been associated with tumor progression and poor survival (40-42). However, this might only apply to inactivated pDC (43-45) as in contrast their activation by Toll-like receptor agonists provided a significant tumor rejection or even complete regression (46-48).

Finally, we aimed to find immune cell compositions that are useful for recognition of individual therapy responses and for estimation of suited time points for inclusion of further therapies. We found two blood withdrawal time points that were very striking and both of them were related to tumor progression. The first one was in week 29 directly after detection of the first relapse. Here, the CD4:CD8 ratio was extremely reversed and we found an elevation of neutrophils, monocytes, and myeloid DCs (mDCs) (Figures S1A,C,D in Supplementary Material). The second one was in week 50 directly before start of the second RCT after detection of the second relapse. Here, we observed a huge increase in neutrophils, as well as an elevation of B cells, eosinophils, and mDCs, and likewise the ratio of CD4:CD8 was reversed (Figure 3; Figure S1 in Supplementary Material).

\section{CONCLUDING REMARKS}

We identified several connections between administration or response to radiochemo(immune)-therapy in GBM and variations in the immune cell compositions in the peripheral blood of the patient presented here. To pin distinct immune modulations to definite clinical responses, the obtained data including those related to the numerous additional immune cell subsets/ parameters that have been determined by our immunophenotyping (13) will be analyzed in future in comparison to that of other patients of the IMMO-GLIO-01 study. We, however, already observed in the presented case modulations of activation markers in dependence on the therapy, such as CD38 $8^{+}$expression on T cells (Figure S2 in Supplementary Material) and C69 expression on B cells (Figure S3 in Supplementary Material). Merging these data will allow us to link immune matrices to clinical responses. Anyhow and importantly, here we identified distinct immune modulations in the peripheral blood on single patient level connected to tumor progression or therapy. This knowledge obtained by detailed and closely-meshed immunomonitoring without exclusion of distinct immune cell subsets by sample preparation might be meaningful for personalized therapy approaches of the future. However, this has to be proven by analyses of more patients within the IMMO-GLIO-01 trial. It might be applied to predict therapy responses or to support the identification of tumor progression. Immune monitoring should focus on liquid biopsies since closely-meshed monitoring is mandatory (see weekly alterations displayed in Figure 3) and liquid biopsies are easy to obtain, even in clinical routine (49). Booster immunization based on DC therapies (50) should be considered when beneficial CD8:CD4 ratio is detected. Since eosinophils orchestrate cancer rejection by normalizing tumor vessels and enhancing infiltration of $\mathrm{CD}^{+} \mathrm{T}$ cells (24) at these time points (in the presented case in weeks 10-18), therapeutically activation of eosinophils might emerge as a promising tool for the improvement of clinical cancer immunotherapy.

\section{ETHICS STATEMENT}

Ethical approval (reference number: 266_13B) was granted from the ethics committee of the Medical School of the FriedrichAlexander-Universität Erlangen-Nürnberg and the study has been performed in accordance with the Declaration of Helsinki. Every patient filled out written informed consent prior to inclusion into the study including consent that the obtained data can be published as case report or as any other publication in a pseudonymised manner.

\section{AUTHOR CONTRIBUTIONS}

PR performed most of the immunophenotyping and most of the data analyses and drafted the manuscript together with UG, BF, and AS. NG performed most of the clinical analyses (imaging data) together with AS and contributed to the writing of the manuscript. RW helped to perform the immunophenotyping and contributed to the data analyses. RF contributed to the design of the work. UG drafted and designed the study together with AS and $\mathrm{BF}$, drafted the manuscript, and wrote it together with PR, AS, and BF. AS drafted and designed the study together with USG and $\mathrm{BF}$, contributed to drafting of the manuscript, and performed the clinical analyses together with NG. BF drafted and designed the study together with UG and AS, drafted the manuscript, contributed to data analyses, and wrote the manuscript together with PR, AS, and UG.

\section{ACKNOWLEDGMENTS}

We thank Dr. Eva-Maria Weiss for contributing to the writing of the ethics application for this study. We further acknowledge the support by the German Research Foundation and the FriedrichAlexander-Universität Erlangen-Nürnberg within the funding program Open Access Publishing.

\section{FUNDING}

This work was funded by the Department of Radiation Oncology of the Universitätsklinikum Erlangen and in part by the German Federal Ministry of Education and Research (BMBF) under Grant 02NUK017G (GREWIS).

\section{SUPPLEMENTARY MATERIAL}

The Supplementary Material for this article can be found online at http://journal.frontiersin.org/article/10.3389/fneur.2017.00296/ full\#supplementary-material. 


\section{REFERENCES}

1. Ostrom QT, Gittleman H, Liao P, Rouse C, Chen Y, Dowling J, et al. CBTRUS statistical report: primary brain and central nervous system tumors diagnosed in the United States in 2007-2011. Neuro Oncol (2014) 16(Suppl 4):iv1-63. doi:10.1093/neuonc/nou223

2. Stupp R, Dietrich PY, Ostermann Kraljevic S, Pica A, Maillard I, Maeder $\mathrm{P}$, et al. Promising survival for patients with newly diagnosed glioblastoma multiforme treated with concomitant radiation plus temozolomide followed by adjuvant temozolomide. J Clin Oncol (2002) 20(5):1375-82. doi:10.1200/ JCO.2002.20.5.1375

3. Stupp R, Mason WP, van den Bent MJ, Weller M, Fisher B, Taphoorn MJ, et al. Radiotherapy plus concomitant and adjuvant temozolomide for glioblastoma. N Engl J Med (2005) 352(10):987-96. doi:10.1056/NEJMoa043330

4. McLendon RE, Halperin EC. Is the long-term survival of patients with intracranial glioblastoma multiforme overstated? Cancer (2003) 98(8):1745-8. doi:10.1002/cncr.11666

5. Louis DN, Ohgaki H, Wiestler OD, Cavenee WK, Burger PC, Jouvet A, et al. The 2007 WHO classification of tumours of the central nervous system. Acta Neuropathol (2007) 114(2):97-109. doi:10.1007/s00401-007-0278-6

6. Fecci PE, Heimberger AB, Sampson JH. Immunotherapy for primary brain tumors: no longer a matter of privilege. Clin Cancer Res (2014) 20(22):5620-9. doi:10.1158/1078-0432.CCR-14-0832

7. Postow MA, Callahan MK, Barker CA, Yamada Y, Yuan J, Kitano S, et al. Immunologic correlates of the abscopal effect in a patient with melanoma. $N$ Engl J Med (2012) 366(10):925-31. doi:10.1056/NEJMoa1112824

8. Bielamowicz K, Khawja S, Ahmed N. Adoptive cell therapies for glioblastoma. Front Oncol (2013) 3:275. doi:10.3389/fonc.2013.00275

9. Ransohoff RM, Kivisakk P, Kidd G. Three or more routes for leukocyte migration into the central nervous system. Nat Rev Immunol (2003) 3(7):569-81. doi:10.1038/nri1130

10. Van Woensel M, Mathivet T, Wauthoz N, Rosiere R, Garg AD, Agostinis P, et al. Sensitization of glioblastoma tumor micro-environment to chemo- and immunotherapy by galectin-1 intranasal knock-down strategy. Sci Rep (2017) 7(1):1217. doi:10.1038/s41598-017-01279-1

11. Eder K, Kalman B. The dynamics of interactions among immune and glioblastoma cells. Neuromolecular Med (2015) 17(4):335-52. doi:10.1007/ s12017-015-8362-x

12. Adamczyk LA, Williams H, Frankow A, Ellis HP, Haynes HR, Perks C, et al. Current understanding of circulating tumor cells - potential value in malignancies of the central nervous system. Front Neurol (2015) 6:174. doi:10.3389/ fneur.2015.00174

13. Ruhle PF, Fietkau R, Gaipl US, Frey B. Development of a modular assay for detailed immunophenotyping of peripheral human whole blood samples by multicolor flow cytometry. Int J Mol Sci (2016) 17(8):1-29. doi:10.3390/ ijms17081316

14. Frey B, Rubner Y, Wunderlich R, Weiss EM, Pockley AG, Fietkau R, et al. Induction of abscopal anti-tumor immunity and immunogenic tumor cell death by ionizing irradiation - implications for cancer therapies. Curr Med Chem (2012) 19(12):1751-64. doi:10.2174/092986712800099811

15. Minniti G, Lanzetta G, Scaringi C, Caporello P, Salvati M, Arcella A, et al. Phase II study of short-course radiotherapy plus concomitant and adjuvant temozolomide in elderly patients with glioblastoma. Int J Radiat Oncol Biol Phys (2012) 83(1):93-9. doi:10.1016/j.ijrobp.2011.06.1992

16. Belka C, Ottinger H, Kreuzfelder E, Weinmann M, Lindemann M, LeppleWienhues A, et al. Impact of localized radiotherapy on blood immune cells counts and function in humans. Radiother Oncol (1999) 50(2):199-204. doi:10.1016/S0167-8140(98)00130-3

17. Ostermann S, Csajka C, Buclin T, Leyvraz S, Lejeune F, Decosterd LA, et al. Plasma and cerebrospinal fluid population pharmacokinetics of temozolomide in malignant glioma patients. Clin Cancer Res (2004) 10(11):3728-36. doi:10.1158/1078-0432.CCR-03-0807

18. Hirose Y, Berger MS, Pieper RO. p53 effects both the duration of G2/M arrest and the fate of temozolomide-treated human glioblastoma cells. Cancer Res (2001) 61(5):1957-63.

19. Hickey MJ, Malone CC, Erickson KE, Gomez GG, Young EL, Liau LM, et al. Implementing preclinical study findings to protocol design: translational studies with alloreactive CTL for gliomas. Am J Transl Res (2012) 4(1):114-26.
20. Grossman SA, Ye X, Lesser G, Sloan A, Carraway H, Desideri S, et al. Immunosuppression in patients with high-grade gliomas treated with radiation and temozolomide. Clin Cancer Res (2011) 17(16):5473-80. doi:10.1158/1078-0432.CCR-11-0774

21. Lotfi R, Lee JJ, Lotze MT. Eosinophilic granulocytes and damage-associated molecular pattern molecules (DAMPs): role in the inflammatory response within tumors. J Immunother (2007) 30(1):16-28. doi:10.1097/01. cji.0000211324.53396.f6

22. Davis BP, Rothenberg ME. Eosinophils and cancer. Cancer Immunol Res (2014) 2(1):1-8. doi:10.1158/2326-6066.CIR-13-0196

23. Gatault S, Legrand F, Delbeke M, Loiseau S, Capron M. Involvement of eosinophils in the anti-tumor response. Cancer Immunol Immunother (2012) 61(9):1527-34. doi:10.1007/s00262-012-1288-3

24. Carretero R, Sektioglu IM, Garbi N, Salgado OC, Beckhove P, Hammerling GJ. Eosinophils orchestrate cancer rejection by normalizing tumor vessels and enhancing infiltration of CD8(+) T cells. Nat Immunol (2015) 16(6):609-17. doi:10.1038/ni.3159

25. Lauber K, Ernst A, Orth M, Herrmann M, Belka C. Dying cell clearance and its impact on the outcome of tumor radiotherapy. Front Oncol (2012) 2:116. doi:10.3389/fonc.2012.00116

26. Kotlan B, Simsa P, Teillaud JL, Fridman WH, Toth J, McKnight M, et al. Novel ganglioside antigen identified by B cells in human medullary breast carcinomas: the proof of principle concerning the tumor-infiltrating B lymphocytes. J Immunol (2005) 175(4):2278-85. doi:10.4049/jimmunol.175.4.2278

27. Nzula S, Going JJ, Stott DI. Antigen-driven clonal proliferation, somatic hypermutation, and selection of B lymphocytes infiltrating human ductal breast carcinomas. Cancer Res (2003) 63(12):3275-80.

28. Dong HP, Elstrand MB, Holth A, Silins I, Berner A, Trope CG, et al. NK- and B-cell infiltration correlates with worse outcome in metastatic ovarian carcinoma. Am JClin Pathol (2006) 125(3):451-8. doi:10.1309/15B66DQMFYYM78CJ

29. Tsou P, Katayama H, Ostrin EJ, Hanash SM. The emerging role of B cells in tumor immunity. Cancer Res (2016) 76(19):5597-601. doi:10.1158/00085472.CAN-16-0431

30. Rubtsov AV, Rubtsova K, Kappler JW, Jacobelli J, Friedman RS, Marrack P. CD11c-expressing B cells are located at the T cell/B cell border in spleen and are potent APCs. J Immunol (2015) 195(1):71-9. doi:10.4049/jimmunol. 1500055

31. Inoue S, Leitner WW, Golding B, Scott D. Inhibitory effects of B cells on antitumor immunity. Cancer Res (2006) 66(15):7741-7. doi:10.1158/0008-5472. CAN-05-3766

32. Shalapour S, Font-Burgada J, Di Caro G, Zhong Z, Sanchez-Lopez E, Dhar D, et al. Immunosuppressive plasma cells impede T-cell-dependent immunogenic chemotherapy. Nature (2015) 521(7550):94-8. doi:10.1038/ nature 14395

33. Lampson LA. Monoclonal antibodies in neuro-oncology: getting past the blood-brain barrier. MAbs (2011) 3(2):153-60. doi:10.4161/mabs.3 2.14239

34. Verhoeff JJ, van Tellingen O, Claes A, Stalpers LJ, van Linde ME, Richel DJ, et al. Concerns about anti-angiogenic treatment in patients with glioblastoma multiforme. BMC Cancer (2009) 9:444. doi:10.1186/1471-2407-9-444

35. Goel S, Duda DG, Xu L, Munn LL, Boucher Y, Fukumura D, et al. Normalization of the vasculature for treatment of cancer and other diseases. Physiol Rev (2011) 91(3):1071-121. doi:10.1152/physrev.00038.2010

36. Dirkx AE, oude Egbrink MG, Castermans K, van der Schaft DW, Thijssen VL, Dings RP, et al. Anti-angiogenesis therapy can overcome endothelial cell anergy and promote leukocyte-endothelium interactions and infiltration in tumors. FASEB J (2006) 20(6):621-30. doi:10.1096/fj.05-4493com

37. Osada T, Chong G, Tansik R, Hong T, Spector N, Kumar R, et al. The effect of anti-VEGF therapy on immature myeloid cell and dendritic cells in cancer patients. Cancer Immunol Immunother (2008) 57(8):1115-24. doi:10.1007/ s00262-007-0441-X

38. Xu Y, Villalona-Calero MA. Irinotecan: mechanisms of tumor resistance and novel strategies for modulating its activity. Ann Oncol (2002) 13(12):1841-51. doi:10.1093/annonc/mdf337

39. Kawahara M. Irinotecan in the treatment of small cell lung cancer: a review of patient safety considerations. Expert Opin Drug Saf (2006) 5(2):303-12. doi:10.1517/14740338.5.2.303 
40. Faget J, Bendriss-Vermare N, Gobert M, Durand I, Olive D, Biota C, et al. ICOS-ligand expression on plasmacytoid dendritic cells supports breast cancer progression by promoting the accumulation of immunosuppressive CD4+ T cells. Cancer Res (2012) 72(23):6130-41. doi:10.1158/0008-5472. CAN-12-2409

41. Jensen TO, Schmidt H, Moller HJ, Donskov F, Hoyer M, Sjoegren P, et al. Intratumoral neutrophils and plasmacytoid dendritic cells indicate poor prognosis and are associated with pSTAT3 expression in AJCC stage I/II melanoma. Cancer (2012) 118(9):2476-85. doi:10.1002/cncr.26511

42. Labidi-Galy SI, Treilleux I, Goddard-Leon S, Combes JD, Blay JY, RayCoquard I, et al. Plasmacytoid dendritic cells infiltrating ovarian cancer are associated with poor prognosis. Oncoimmunology (2012) 1(3):380-2. doi:10.4161/onci.18801

43. Beckebaum S, Zhang X, Chen X, Yu Z, Frilling A, Dworacki G, et al. Increased levels of interleukin-10 in serum from patients with hepatocellular carcinoma correlate with profound numerical deficiencies and immature phenotype of circulating dendritic cell subsets. Clin Cancer Res (2004) 10(21):7260-9. doi:10.1158/1078-0432.CCR-04-0872

44. Treilleux I, Blay JY, Bendriss-Vermare N, Ray-Coquard I, Bachelot T, Guastalla JP, et al. Dendritic cell infiltration and prognosis of early stage breast cancer. Clin Cancer Res (2004) 10(22):7466-74. doi:10.1158/10780432.CCR-04-0684

45. Vermi W, Bonecchi R, Facchetti F, Bianchi D, Sozzani S, Festa S, et al. Recruitment of immature plasmacytoid dendritic cells (plasmacytoid monocytes) and myeloid dendritic cells in primary cutaneous melanomas. J Pathol (2003) 200(2):255-68. doi:10.1002/path.1344

46. Le Mercier I, Poujol D, Sanlaville A, Sisirak V, Gobert M, Durand I, et al. Tumor promotion by intratumoral plasmacytoid dendritic cells is reversed by
TLR7 ligand treatment. Cancer Res (2013) 73(15):4629-40. doi:10.1158/00085472.CAN-12-3058

47. Preynat-Seauve O, Schuler P, Contassot E, Beermann F, Huard B, French LE. Tumor-infiltrating dendritic cells are potent antigen-presenting cells able to activate T cells and mediate tumor rejection. J Immunol (2006) 176(1):61-7. doi:10.4049/jimmunol.176.1.61

48. Liu C, Lou Y, Lizee G, Qin H, Liu S, Rabinovich B, et al. Plasmacytoid dendritic cells induce NK cell-dependent, tumor antigen-specific T cell cross-priming and tumor regression in mice. JClin Invest (2008) 118(3):1165-75. doi:10.1172/JCI33583

49. Quandt D, Zucht HD, Amann A, Wulf-Goldenberg A, Borrebaeck C, Cannarile $\mathrm{M}$, et al. Implementing liquid biopsies into clinical decision making for cancer immunotherapy. Oncotarget (2017). doi:10.18632/oncotarget.17397

50. Burdach SE, Bliesener JA, Evers KG, Gharib M. [Pre- and retrorenal neuroblastoma without kidney displacement]. Klin Padiatr (1983) 195(1):52-6. doi:10.1055/s-2008-1034041

Conflict of Interest Statement: The authors declare that the research was conducted in the absence of any commercial or financial relationships that could be construed as a potential conflict of interest.

Copyright (c) 2017 Rühle, Goerig, Wunderlich, Fietkau, Gaipl, Strnad and Frey. This is an open-access article distributed under the terms of the Creative Commons Attribution License (CC BY). The use, distribution or reproduction in other forums is permitted, provided the original author(s) or licensor are credited and that the original publication in this journal is cited, in accordance with accepted academic practice. No use, distribution or reproduction is permitted which does not comply with these terms. 